\title{
The Solar-Stellar Connection and Disconnection
}

\author{
Klaus G. Strassmeier \\ Astrophysical Institute Potsdam (AIP), An der Sternwarte 16, D-14482 \\ Potsdam, Germany
}

\begin{abstract}
The study of stellar activity is now an almost classical astronomical topic. The first Ca II-H\&K observations were made a hundred years ago by Eberhard \& Schwarzschild ${ }^{1}$ and many thousand papers were published after its rediscovery some three decades ago by $\mathrm{O}$. C. Wilson. The complexity of the atmospheric and interior magnetic activity as observed on the Sun is hard, if not impossible, to extrapolate to solar-type stars. So far there is no solar twin found, despite that it appears that just a single process acts as the driving mechanism for activity in all atmospheric layers and partially even in the convective envelope: the dynamodriven magnetic field. In this paper, I will try to give examples where the solar analogy holds and where it is clearly not appropriate, putting some emphases on differential surface rotation and meridional circulation. I stress the importance of mapping stellar surfaces as fingerprints of the underlying dynamo action and directly measure surface magnetic fields.
\end{abstract}

\section{To understand a simple thing as a star...}

We still chew on this phrase from Arthur Eddington's famous book and, despite of a now three decades long modern publication record of exploring stellar evolution beyond the Voigt-Russell theorem, there are still many basic unanswered questions, and more are yet to come. My aim in this paper is to point out the importance of the stellar magnetic field for stellar evolution of late-type stars in general, for the surfaces of the Sun and solar-type stars in special. Magnetic fields and their geometry are usually not the driver for evolutionary processes in stellar evolution but again, neglecting its presence may lead to imprecise and even false models. After all, magnetic flux must be preserved throughout the history of a star or a star cluster, starting during the complex star formation phase, the subsequent stellar contraction and accretion phase, its main-sequence time and, more and more obvious, also in the late stages on the AGB and possibly also in its planetary nebulae phase. It may actually turn out that it is the current evolutionary stage of the Sun that is to be the least affected by magnetic fields, despite that we use it as our model star for everything.

\footnotetext{
${ }^{1}$ Eberhard \& Schwarzschild (1913) discovered CaII H\&K emission, e.g., in the spectrum of
} $\sigma$ Gem. 
One key question for solar-stellar astrophysicists is to prove that the solar phenomena are indeed typical for solar-type stars. In other words, can we compare the Sun with other stars in the first place? Aren't slight differences in mass, effective temperature and metallicity reason enough that the star takes a different route in the development of its magnetic field geometry and thus its angular-momentum loss? Obviously, we are dealing with a multi-component parameter space with cross-connected and back-reacting entities, as is usual in magnetohydrodynamics. In this respect it is no surprise that we had not found the sought for solar twins yet. Or maybe we are still thinking too simple minded. Let's remember Sir Arthur again.

\section{Where are the solar twins?}

\subsection{The sample: our galaxy}

Extrapolated number counts based on various sample directions suggest a total of up to 400 billion stars in our galaxy. Detailed extrapolation from the local neighborhood hints towards a total of $\approx 26$ billion $\mathrm{G}$ dwarfs in our galaxy of which roughly one billion is expected to be of spectral classification G2V. Therefore, there should be plenty of Sun's around us, but apparently there are not. Based on the Hipparcos distances for $\approx 100,000$ stars together with the Tycho catalog for $\approx 2$ million stars, we can only search the solar backyard. The result is annoying: In a volume of radius $12.5 \mathrm{lyr}$ there is only one additional star that has been classified G2V, i.e. $\alpha$ Cen A (it also happens to be the second nearest after Proxima Centauri, the third component in the wide triple system $\alpha$ CenAB see later). The total number of stars in that volume is 33 , made up almost exclusively by M- and K-dwarfs.

The "Nstars"-project (Henry et al. 1997) aims to reach completeness out to 5 pc (16 lyr), now expanded to 25 pc (Henry et al. 2002). The recent discovery of the nearest brown dwarf at 6 lyr ( $\epsilon$ Ind B; Scholz et al. 2003 ${ }^{2}$ ) suggests that this goal is not yet reached. Henry et al. extrapolated the cumulative number of stars within the $5 \mathrm{pc}$ of Nstars out to $10 \mathrm{pc}$ and compared that to actually observed number counts and found a total number of 130 missing systems. If we continue in our search for G2V stars in the Hipparcos/Tycho sample and enlarge the search volume to a radius of $20 \mathrm{lyr}(\approx 6 \mathrm{pc})$, we still find only $\alpha$ Cen $\mathrm{A}$ as the only other G2V star out of 111 stars in a total of 79 stellar systems. Increasing the search radius to 50 lyr $(\approx 15 \mathrm{pc})$ there are 1800 stars in 1200 stellar systems, with a total of $5 \mathrm{G} 2 \mathrm{~V}$ stars including $\alpha$ Cen. Finally, going out to a distance of 250 lyr ( $\approx 77 \mathrm{pc}$ ), we expect 250,000 stars, but then we lack precise spectral classifications and parallaxes of G2V-stars in the Hipparcos catalog.

\subsection{Best examples so far}

Hardorp (1978) studied 77 solar-type stars and considered 10 "solar analogs" in detail but classified only one star "indistinguishable from solar": HD44594. At the time of his study, no information on $[\mathrm{Fe} / \mathrm{H}]$ was available. It soon turned out

\footnotetext{
${ }^{2}$ Recently discovered to be a binary brown dwarf. This discovery was made independently at the ESO-VLT (McCaughrean et al. 2003) and at Gemini-South (Walker et al. 2003).
} 
that HD44594 is 0.15dex overabundant in metals as compared to the Sun. Nevertheless, it was Johannes Hardorp who carried out the first systematic searches for solar analogs and motivated a number of subsequent studies. E.g., Dorren \& Guinan (1994) listed 11 G0-G5 "solar proxies", including $\beta$ Hyi, an old G2IV star, that nowadays is the best studied proxy for the "old" Sun. Gray (1995) applied the line-ratio technique to a large number of stars to determine very precise relative photospheric temperatures. He found 6 stars within $20 \mathrm{~K}$ of the nominal solar value of $5770 \mathrm{~K}$ : HR4345, 18 Sco, HR8314, 16 CygA, 26 Dra, and $\pi^{1}$ UMa. The range of metallicity for these candidates is $[\mathrm{Fe} / \mathrm{H}]=-0.06$ to +0.04 . The range in rotational velocity, $v \sin i$, is between 0 (unresolvable at even high resolution) and $9 \mathrm{~km} / \mathrm{s}$. About at the same time, Cayrel de Strobel's (1996) seminal review of solar-analogues appeared and marked the then state of the art. His work showed that out of 105 photometric solar analogs, only 5 remained with the three parameters $T_{\text {eff }}, M_{\text {bol }}$, and $[\mathrm{Fe} / \mathrm{H}]$ approximately solar. In his conclusions, he then favored 51 Peg (G2.5) and HD76151 (G3) to be the best solar analogues around. Note that at the time of the writing of his review, the planet of 51 Peg was not yet known (but see the "note added in press" added at the end of his review).

Many more papers appeared thereafter and further increased and detailed the data base. Lockwood et al. (1997) photometrically monitored a large number of late-type stars, but out of 41 "sun-like" stars only 7 were G0-3 single stars. Gaidos (1998) published the first catalog of 38 "solar analogs" within 25 pc. His "analogy parameter", $A$, is solely based on mass and luminosity and is aimed towards a quantification of the term "solar analog": $A=(L / 0.69) M^{-2 / 3}$. The typical range in his catalog is $2 / 3<A<3 / 2$ (obviously, $A=1$ is the perfect twin, which is never reached in the catalog).

More recently, the search for solar twins with high-resolution spectroscopy even includes some open clusters. E.g., Barrett et al. (2001) presented a list of 14 "solar twins" in M67. Somewhat earlier, a first scientific meeting was dedicated to the question of solar analogs (Hall 1997). The outcome of the discussions of this meeting was that 18 Sco may be the best "solar-twin" so far (R. Garrison, private communication).

In the meantime, Gaidos \& Gonzales (2002) conducted high-resolution optical follow-up spectroscopy of the Gaidos (1998) sample and redetermined more precise spectral classifications, metallicities etc.. Follow-up photometry of 34 out of the 38 stars was already presented by Gaidos et al. (2000). Quite disappointing though, only a handful of stars were left as serious candidates for real solar twins. As a matter of fact no star was classified as G2 in their sample. The remaining sample consisted of 1 G1.5 star ( $\left.\pi^{1} \mathrm{UMa}\right), 1 \mathrm{G} 1 \mathrm{star}$ (HD130948), 2 G3 stars (HD1835, HD30495), 1 G4 star (HD59967), and 5 G0 stars.

Finally, even the last resort of hope was pulverized by the VLTI: $\alpha$ Cen A, classified as G2V. Kervella et al. (2003) spatially resolved $\alpha$ Cen A and B during a run with the VINCI commissioning instrument on the VLTI and determined precise absolute radii based on the Hipparcos distance. $\alpha$ Cen A is $50 \%$ more luminous than the Sun despite that the effective temperature differs only by $20 \mathrm{~K}$ and the star appears to be only $\approx 200$ million years older. 


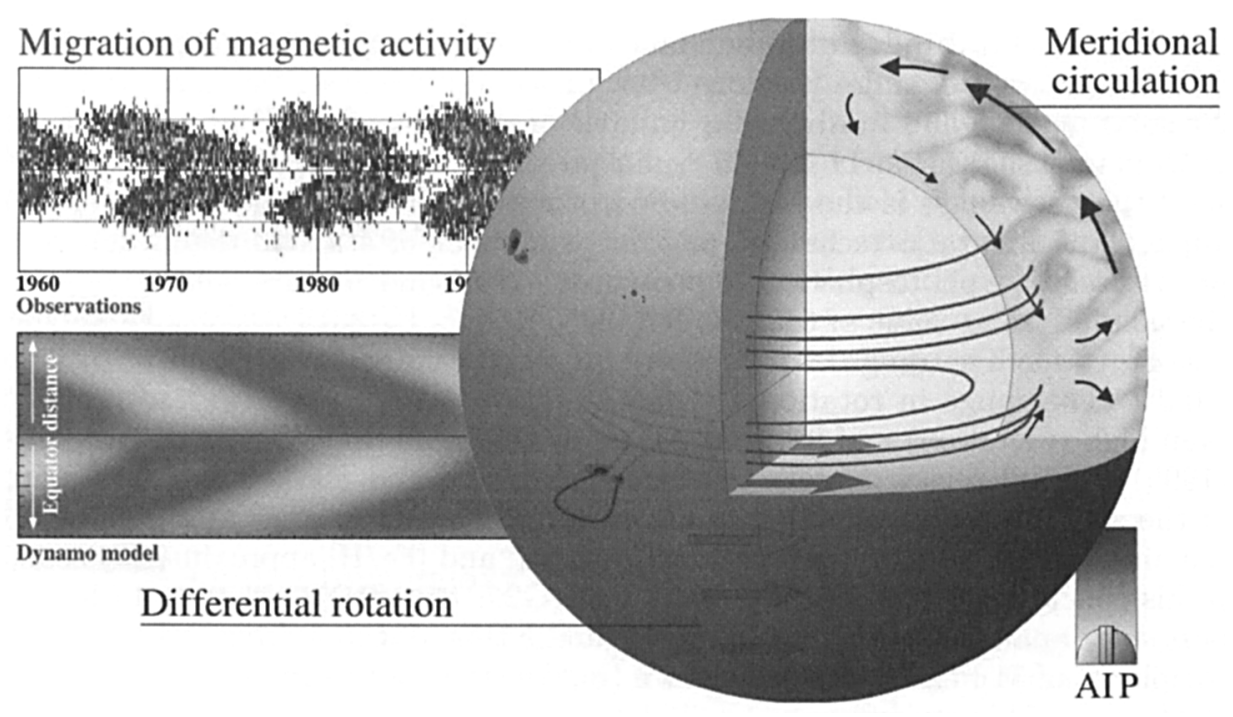

Figure 1. The link between surface activity and internal dynamo action. Magnetic flux will be produced by a dynamo driven by convective motions at the bottom of the convection layer, by differential rotation and by meridional flows. The differential rotation winds up the dipole magnetic field and converts it into one mainly in the direction of rotation (curved thin lines). At the bottom of the convection zone, this field is transported towards the equator by a meridional flow. When single flux-tubes of this field become unstable they will rise to the surface and eventually cause starspots.

\subsection{A solar-stellar disconnection?}

Is the fact that we have such troubles finding a solar twin a sign of a solar-stellar disconnection? Or in other words, what do we learn from the fact that stars with solar parameters are so rare. Or aren't they rare at all and we just haven't looked deep enough? Academically it is very interesting to explore the possibility that stars are individuals, in spite that current star-formation theories suggest that stars form preferably in clusters of hundreds or even thousands of stars and not as single field stars (Klessen 2003).

\section{4. $\quad \ldots$ and possible reconnections!}

However, help is on the horizon in form of three space missions. The NASA mission Kepler will conduct a photometric monitoring program of almost 100,000 G-K stars in a field in the Cygnus region. Its launch is foreseen for late 2007. Maybe only a few months later, the ESA mission Eddington will - hopefully - follow in early 2008 and conduct an even wider science program including asteroseismology. Finally, ESA's GAIA mission will provide the fundamental stellar data for a billion stars out to $20 \mathrm{kpc}$ by 2015 or so. A few years later is the time we probably have the final answer for the solar-analog issue. 


\section{Surface activity and dynamo}

\subsection{Is there a single relation?}

Frazier (1972) and others had shown that the Ca II emission-line flux from the Sun increases with photospheric magnetic field strength. Subsequently, this was demonstrated for many more spectral features (a brief summary of these efforts is given by Schrijver 1996). So, at first glance, above question has an obvious answer. Yes, atmospheric emission-line flux is related to the surface magnetic field. But the question is, how strongly and in what way is the surface magnetic field, or magnetic flux, related to the internal dynamo? For any star other than the Sun we have only qualitative statements, because the evidence is based on integral measures of stellar surface activity, i.e. disk-integrated measurement of, e.g., Ca II emission or broad-band UV enhancement. The most detailed data we have are from stars rotating several times to tens of times faster than the Sun. Some surprises were found, e.g., that there is a "saturation" of the emission-line flux (e.g., for the X-ray flux) when the magnetic filling factor of the atmospheric volume comes close to $100 \%$.

Obviously, what we need are observations that spatially resolve the stellar disk and provide evidence for the existence of a systematically changing pattern of the magnetic flux, like the solar butterfly diagram. This is the "direct" approach. Unfortunately, it is still in an infant state and involves the successive solution of three major obstacles for observational techniques; firstly, we need to spatially resolve the stellar disk, secondly, we need to distill the geometric effect of the magnetic surface field and, thirdly, we need to do this over the period of an activity cycle. The latter may not appear as an acceptable proposal for a telescope time allocation committee.

What hard observational evidence is there that indeed supports such a relation between dynamo action and surface activity? Fig. 1 tries to summarize the solar paradigm. The joint action of differential rotation (the $\Omega$-effect) and helical turbulence (the $\alpha$-effect) reproduces the observed butterfly diagram. The internal structure and the dynamo process itself act as the tuneable models to come up with the correct surface quantities. The "indirect" stellar approach is now to verify solar surface activity tracers on other stars and then find the "direct" link between the surface and the interior for the Sun, e.g., from helioseismic measurements. This is the classical solar-stellar connection approach, where the Sun acts as the one-and-only model star. But what if the Sun is special?

\subsection{Interface-layer dynamo or distributed dynamo?}

The location of the solar dynamo is most likely associated with a region with sharply increased speed of sound at a depth of roughly $240,000 \mathrm{~km}\left(R \approx 0.66 \mathrm{R}_{\odot}\right)$ and a width of roughly $50,000 \mathrm{~km}$, just below the bottom of the convective envelope (see, e.g., Charbonneau et al. 1999). Previously nicknamed the "overshoot" region, it is a superadiabatically stratified layer in which magnetic fields can reside for long enough and yet experience enough plasma motion from the top to run the dynamo in the first place. This region is nowadays better known as the "tachocline" because it is the shear forces from the differential rotation gradient which is largest in this layer and thus enables the appropriate dynamo action. More recent helioseismic data (e.g., Basu 1997, Basu et al. 2003) suggest a 
smaller thickness of the solar overshoot layer than what was originally claimed (and it is getting thinner!; see Ziegler \& Rüdiger 2003). The ultimate stellar approach would be to verify this from asteroseismology of solar-type stars and, even better but further into the future, from spatially resolved asteroseismology. This is - by the way - exactly the goal of the Stellar Imager (SI), a proposed free-flying UV interferometer with spectral dispersion for 2020 or so (Carpenter et al. 2003) .

However, an interface-layer dynamo is not the only possibility. The rapidlychanging cell structure of solar granules may be interpreted as the host of many small distributed dynamos just below the $\tau=1$ surface (Cattaneo 1999). This is seen in 3-D numerical MHD simulations of the solar granulation and also even for M supergiants (Freytag et al. 2002). The tendency to form rolls instead of cells once rotation and a weak magnetic field is included (Ziegler 2002) is even further support for a distributed dynamo. If correct, it is likely that the observed, weaker and spatially more distributed fields, like the magnetic-field carpet of the chromospheric network, are due to such a surface-layer dynamo while larger features, possibly as small as pores and as large as full active regions, are driven by the interface-layer dynamo from deep underneath. The agent to transport the magnetic flux up to the surface is still our classical flux tube according to Parker (1955). Together with a typical field strength of $10 \mathrm{~T}$, the diameter of a flux tube becomes about $2000 \mathrm{~km}$ in the overshoot layer, but increases strongly as the tube rises through the convection zone. See, e.g., Granzer (2002) for a more detailed discussion of flux-tube emergence in cool stars. The transportation agent through the photosphere, i.e. from just below the $\tau=1$ surface into the lower chromosphere, is likely different. There are some suggestions that it is due to Alfvén waves, but this clearly needs further thinking in the light of the recent modelling success of the chromospheric energy budget with propagating acoustic waves and their interferences (Wedemeyer et al. 2002).

Further evidence for the existence of two types of dynamos comes from the rotational evolution of late-type stars. Just recently, Barnes (2003) explained the bimodality of rotational periods of cool stars, as observed in a number of open clusters with ages between 30 Myrs to 4.5 Gyrs, by means of the two types of dynamo location (see his paper for further references). The slower rotators should posses an interface-layer dynamo like the Sun that connects the radiative interior with the convective envelope as well as with the stellar exterior. These stars will loose angular momentum via a stellar wind and spin down Skumanich-style. The rapidly-rotating very-young G-K rotators, on the other hand, should posses a distributed dynamo which is inefficient to produce a strong large-scale magnetic field in order to lock to the radiative zone, resulting in only an age-dependent spin down of the outer envelope. Eventually, these stars will evolve into the interface-layer regime by locking in onto the radiative zone. Fully convective $M$ stars can never run an interface-layer dynamo and thus will never transit within the period bimodality and thus spin down on a very much longer timescale, in agreement with observations (Delfosse et al. 1998).

But again, what if the dynamo of the Sun is special? Although this is easily possible due to the complex physical situation in and below a convection zone - together with the many "tuneable" parameters of any dynamo model - 


\section{Differential rotation of active main-sequence stars}

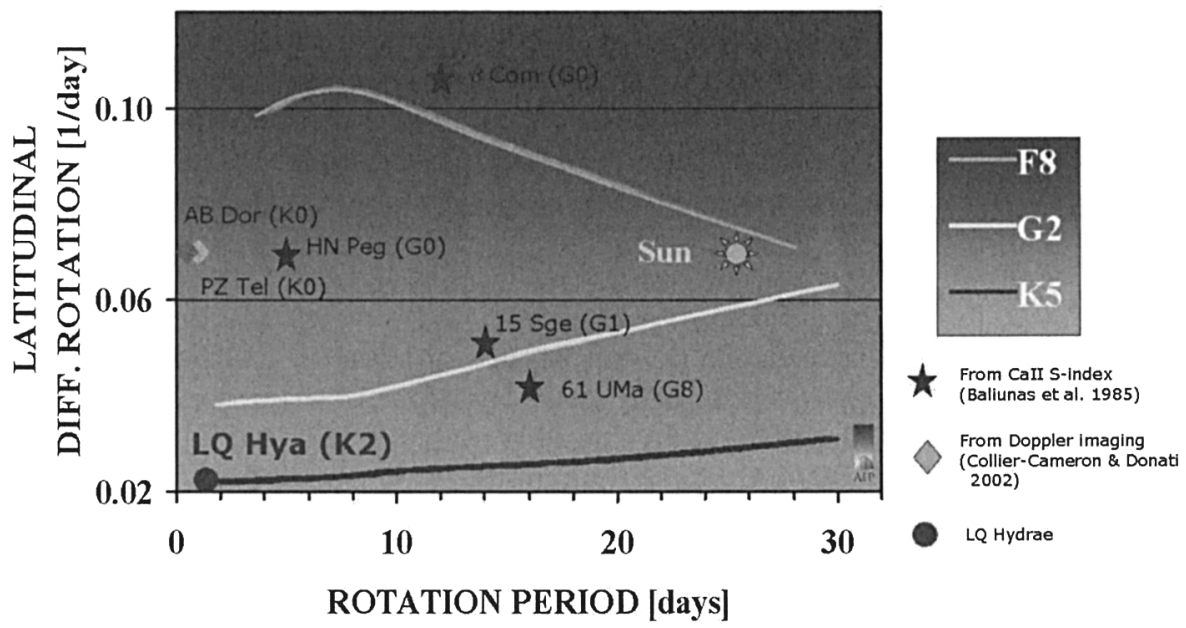

Figure 2. A comparison of measurements of surface differential rotation of active main-sequence stars with theoretical predictions. Data are from Doppler-imaging techniques (diamonds, dots) and Ca II H\&K time-series analysis (stars). The LQ-Hya detection is from Kövári et al. (2003). The Sun is also shown. The lines are models based on an extension of the original work of Kitchatinov \& Rüdiger (1999) and Küker \& Stix (2001).

there is no "hard" evidence so far that other solar-type stars run qualitatively different dynamos. However, there is plenty of indirect evidence that there are some significant differences to the Sun; e.g., the existence of large polar starspots (e.g., Strassmeier 2002), the detection of azimuthal fields near the polar regions (Donati 1999), anti-solar differential rotation (Vogt \& Hatzes 1996) and possibly cyclic-dependent differential rotation (Collier-Cameron \& Donati 2002), or the pure existence of magnetic fields on fully convective low-mass M dwarfs (JohnsKrull \& Valenti 1996) and also of flares on brown dwarfs (Drake et al. 1996, a.o.).

Clearly, after all, having the Sun as a guide is rather invaluable. Whether stellar magnetic activity turns out to be a scaled version of the Sun's activity or something completely different is still to be determined.

\subsection{Activity cycles}

Based on the solar-dynamo paradigm, one could consider the pure detection of an activity cycle "medium-hard" evidence for the existence of a relation between the stellar interior and the surface. The Mt. Wilson H\&K program has revealed cycle periods of "solar-type stars" in the range 3-21 years (Baliunas et al. 1995). The longer periods are likely not due to a physical limit but rather due to the (still) limited observational baseline (after 30 years of continuous 
observations!). Many stars are known with H\&K-flux trends that either suggest a period in excess of this upper limit or a non-periodic behavior (for updates see the Mt. Wilson-Observatory homepage). Similar is true for continuum photometry where periods of up to 60 years were found from photographic plates (Hartmann et al. 1981) as well as "trends" that may suggest even longer periods (see, e.g., Fröhlich et al. 2004, Oláh \& Strassmeier 2002, Saar \& Brandenburg 1999). The shorter cycle lengths are even more interesting to interpret (but not here because I run out of space).

\subsection{Differential rotation and meridional circulation}

Fig. 1 had already emphasized the importance of these two phenomena for the solar butterfly diagram. While differential rotation, both in depth and on the surface, causes shear forces that can change a poloidal field into a toroidal field it is the meridional circulation that can contribute to the opposite effect, i.e. changing parts of the toroidal component back into a poloidal component. Therefore, detecting these two phenomena - differential rotation and meridional circulation - on other stars would place tight constraints on the type of dynamo in the stellar interior.

Differential stellar rotation was investigated by several authors as early as the 1950's (e.g., Slettebak 1949, Huang 1961) but the time was not ripe due to the too low a quality of the spectral data. But even detections from modern high-quality high-resolution spectral line observations with the help of, e.g., Fourier Transform techniques were mostly negative or inconclusive (Gray 1977, Gray \& Baliunas 1997), while the detections from long-term H\&K-photometry have the problem that a 2-D phenomenon is concluded from 1-D observations, and is considered ambiguous (e.g., Donahue \& Baliunas 1992). Probably the first indirect detection of differential rotation on an active late-type star was for the K subgiant of the RS CVn binaries UX Ari and HR 1099 by Vogt \& Hatzes $(1991,1996)$. Vogt \& Hatzes had used consecutive Doppler images to follow the longitudinal migration of individual spots and then reconstructed the surface differential rotation law. Much to everybody's surprise, the sign of the differential rotation was antisolar, i.e. the poles were rotating faster than the equator; but see Petit et al. (2003) for a contradictory result. Later on, Donati \& Collier-Cameron (1997) and Weber \& Strassmeier (1998) had followed up with a cross-correlation method and applied it to AB Doradus, a K-type ZAMS star, and to IL Hydrae, a G8 subgiant, respectively. Many papers followed and the search for differential rotation on a variety of stars, old and young, solar and non-solar, is still going on (see Table 1; the table is an updated version of the one in Korhonen 2002). In this Table, the columns are as follows: "Method" indicates the method: $\mathrm{ph}=$ photometry, DI=Doppler imaging $(\mathrm{ST}=$ spot tracking, $\mathrm{CCF}=$ cross correlation, $\mathrm{SI}=$ sheared image, $\mathrm{ZDI}=\mathrm{Zeeman}-$ DI), $\mathrm{Ca}=\mathrm{Ca}$ II H\&K S-index photometry. $\alpha=\Delta \Omega / \Omega$ for DI and ph, while the percentage difference between two periods is given for Ca. "n.det." means not detected; "yes" means detected but not quantifiable.

It is now agreed that differential rotation is a key parameter for understanding stellar activity. Figure 2 compares observational and theoretical findings for rotational-period variations on the stellar surface as a function of the rotational period and spectral class. The symbols mark the observations for 8 active stars. 
Table 1. Detections of differential rotation for active late-type stars. (See text for explanation of column items.)

\begin{tabular}{|c|c|c|c|c|}
\hline Name & Type & Method & $\alpha$ & Reference \\
\hline Sun & single dwarf & various & +0.19 & various \\
\hline UX Ari & RS CVn & DI-ST & -0.020 & Vogt \& Hatzes (1991) \\
\hline V410 Tau & single WTTS & DI-ST & yes & Rice (2002) \\
\hline \multirow[t]{4}{*}{ HR1099 } & RS CVn & DI-ST & -0.0035 & Vogt et al. (1999) \\
\hline & & ph. & 0.006 & Henry et al. (1995) \\
\hline & & DI-CCF & n.det. & Strassmeier \& Bartus (2000) \\
\hline & & DI-ST & yes $^{1}$ & Strassmeier \& Bartus (2000) \\
\hline EI Eri & RS CVn & DI-CCF & -0.1 & Washuettl et al. (2004) \\
\hline HD 31993 & single giant & DI-CCF & -0.12 & Strassmeier et al. (2003) \\
\hline \multirow[t]{2}{*}{ AB Dor } & ZAMS & DI-SI & $+0.046-0.058$ & Cameron \& Donati (2002) \\
\hline & & ZDI & $+0.06-0.1$ & Cameron \& Donati (2002) \\
\hline HD 37824 & RS CVn & $\mathrm{ph}$ & 0.08 & Hall et al. (1991) \\
\hline CM Cam & RS CVn & DI-CCF & n.det. & Strassmeier et al. (1998) \\
\hline \multirow[t]{2}{*}{$\sigma \mathrm{Gem}$} & RS CVn & $\mathrm{ph}$ & 0.038 & Henry et al. (1995) \\
\hline & & DI-CCF & $<+0.004$ & Kővári et al. (2001) \\
\hline IL Hya & RS CVn & DI-CCF & +0.004 & Weber \& Strassmeier (1998) \\
\hline HD 82443 & ZAMS & $\mathrm{ph}$ & +0.04 & Messina et al. (1999) \\
\hline \multirow[t]{2}{*}{ LQ Hya } & ZAMS & DI-SI & $-0.05-+0.2$ & Donati et al. (2003) \\
\hline & & DI-CCF & +0.022 & Kővári et al. (2003) \\
\hline $61 \mathrm{UMa}$ & single dwarf & $\mathrm{Ca}$ & $10 \%$ & Baliunas et al. (1985) \\
\hline HU Vir & RS CVn & DI-ST & -0.011 & Strassmeier (1994) \\
\hline RS CVn & RS CVn & $\mathrm{ph}$ & $0.01-0.04$ & Rodonò et al. (1995) \\
\hline$\beta \mathrm{Com}$ & single dwarf & $\mathrm{Ca}$ & $21 \%$ & Baliunas et al. (1985) \\
\hline \multirow{2}{*}{ FK Com } & FK Com & DI-ST & +0.016 & Korhonen et al. (2002) \\
\hline & & DI-ST & +0.025 & Korhonen et al. (2003) \\
\hline V815 Her & RS CVn & $\mathrm{ph}$ & 0.184 & Jetsu et al. (2000) \\
\hline PZ Tel & ZAMS & DI-CCF & +0.0113 & Barnes et al. (2000) \\
\hline HR 7275 & RS CVn & $\mathrm{ph}$ & $0.022-0.04$ & Strassmeier et al. (1994) \\
\hline 15 Sge & single dwarf & $\mathrm{Ca}$ & $11 \%$ & Baliunas et al. (1985) \\
\hline \multirow[t]{3}{*}{ HD 199178} & FK Com & DI-CCF & n.det. & Strassmeier et al. (1999) \\
\hline & & DI-ST & -0.17 & Hackman et al. (2001) \\
\hline & & DI-ST & -0.11 & Hackman (2003) \\
\hline HN Peg & single dwarf & $\mathrm{Ca}$ & $5 \%$ & Baliunas et al. (1985) \\
\hline \multirow[t]{2}{*}{ RT Lac } & RS CVn & $\mathrm{ph}$ & $0.0032^{2}$ & Lanza et al. (2002) \\
\hline & & & $\sim 0.0027^{3}$ & Lanza et al. (2002) \\
\hline KU Peg & RS CVn & DI-CCF & $+0.09-0.34^{4}$ & Weber \& Strassmeier (2001) \\
\hline$\lambda$ And & RS CVn & $\mathrm{ph}$ & 0.04 & Henry et al. (1995) \\
\hline LO Peg & single dwarf & DI-CCF & n.det. & Lister et al. (1999) \\
\hline \multirow[t]{2}{*}{ IM Peg } & RS CVn & DI-CCF & -0.04 & Weber (2003) \\
\hline & & DI-SI & -0.02 & Weber (2003) \\
\hline \multirow[t]{4}{*}{ II Peg } & RS CVn & $\mathrm{ph}$ & 0.005 & Henry et al. (1995) \\
\hline & & $\mathrm{ph}$ & 0.015 & Rodonò et al. (2000) \\
\hline & & DI-CCF & -0.05 & Weber (2003) \\
\hline & & DI-SI & -0.05 & Weber (2003) \\
\hline LQ Lup & ZAMS & DI-CCF & 0.0059 & Donati et al. (2000) \\
\hline UZ Lib & RS CVn & $\mathrm{ph}$ & -0.0026 & Oláh et al. (2003) \\
\hline Sz68 & single CTTS & DI & maybe & Johns-Krull \& Hatzes (1997) \\
\hline
\end{tabular}

\footnotetext{
${ }^{1}$ pole-ward migration detected but only partially due to differential rotation.
}

${ }^{2}$ primary component. ${ }^{3}$ secondary component. ${ }^{4}$ lower/upper limit. 
The lines show theoretical predictions of a series of papers by Rüdiger, Kitchatinov, Küker, and Stix. The slopes of the theoretical lines change from $\mathrm{K}$ through $\mathrm{G}$ to $\mathrm{F}$ stars. Rapidly rotating, young $\mathrm{F}$ stars are predicted to possess much stronger differential rotation compared to later spectral classes. Common or not common among solar-type stars, the basic question of what drives the differential rotation in the first place still remains to be answered but the connection to the meridional flow seems obvious, and often encountered as the $\alpha^{2}$-effect (see, e.g., Rüdiger \& Hollerbach 2004).

For the hotter and more rapidly-rotating stars, a recent survey of differential rotation on F-star by Reiners \& Schmitt (2003) detected differential rotation on just 10 stars out of a total of 142 targets. Their (projected) lap times exceeded the solar one by several factors though. Despite that the observations require excellent data of ultrahigh spectral resolution, differential rotation may after all be not as common as originally thought, in agreement with some theoretical "predictions". A recent numerical MHD simulation of differential rotation for a T Tauri-like star (Arlt \& Rüdiger 2003) showed that the magnetic field very quickly flattens the velocity gradient and very effectively suppresses the differential rotation (an animated movie can be found at www.aip.de/highlight_archive/arlt_diffrot/). A similar results was found for Cp stars (Arlt et al. 2003).

Meridional circulation is an even more difficult a phenomenon to detect, even for the Sun. Old sunspot observations dating back a century proved the key for its original detection by Tuominen (1942). Howard \& Gilman (1986) and others used well defined small sunspots as tracers and detected systematic poleward migration on the order of a few $\mathrm{m} / \mathrm{s}$ while, at the same time, some equator crossers were detected and tracked. A sound and unified theoretical explanation for its global character is still missing though.

As for differential rotation, Doppler images may provide evidence for meridional circulation on stars other than the Sun. Vogt et al. (1997) and Strassmeier $\&$ Bartus (2000) had found systematic poleward migration of spots on the active sub-giant component of HR 1099 from time series of Doppler images. The latter authors found a value of $0.4^{\circ}$ day. Surprisingly, Weber \& Strassmeier (2001) detected the very same value for the K-giant KU Pegasii, also a component of a close binary system. These star's interior momentum distribution is expected to be very different from the Sun's and thus not directly comparable. Nevertheless, we have now some first evidence for systematic velocity fields on the stellar surface of active stars but we do not know whether these are due to meridional circulation or simply related to unresolved spot motions. More systematic work at better temporal and spectral (=spatial) resolution is to be done.

\subsection{The diversity of stellar-surface morphology}

One of the most stimulating results for the discussion of the solar-stellar connection was the discovery of polar starspots twenty years ago (Vogt \& Penrod 1983). Because the Sun does not have spots at latitudes much in excess of $\pm 45^{\circ}$, we may speculate that stars with polar spots may have a qualitatively different dynamo mechanism. However, it soon turned out that the stars with polar spots do not form a recognizable stellar group with one or more astrophysical 
parameters in common. Nor seems there to be a relation between surface spot morphology and, e.g., stellar rotational period (or velocity).

The papers at the 1st Potsdam Thinkshop on "Sunspots \& Starspots" summarized the situation in 2002, and I refer the reader to its proceedings for a general overview (Strassmeier, Washuettl \& Schwope 2002). One of the many remaining questions is whether the huge spots seen on some of the more active stars are indeed monolithic, with or without a solar-like penumbra, or consist of many smaller spots. The observed log-normal distribution of (umbral) sunspot sizes suggests the latter scenario, if the Sun were observed as a star (Bogdan et al. 1988).

A summary of all Doppler images was given by Strassmeier (2002) and our group in Potsdam maintains a web page at www.aip.de/groups/activity/ that shows IDL-plots of all our Doppler images from the "stellar surface structure" program. These maps can be arranged on the screen according to various stellar parameters e.g., rotation period, spectral type, evolutionary state. Fig. 3 shows an example where we plot one map for one hemisphere per star as a function of rotational period for pre-main-sequence, main-sequence, and evolved stars, respectively. Fig. 3 reveals no obvious dependency of the surface morphology, e.g., a tendency that polar spots appear preferentially on short-period stars, as one may have thought from an extrapolation of the solar-stellar connection. However, the sample not only mixes binaries and single stars, but also covers a range of -0.5 to +0.2 dex in elemental abundance with respect to the Sun and, as a technicality, used either $\mathrm{Ca}$ or Fe lines for the line-profile inversion.

\subsection{From stars to the Sun: active longitudes and flip-flops}

Active longitudes, i.e. sections of preferred spot locations, were first seen for the spotted components of several of the short-period RS CVn-type binaries by Zeilik et al. (1983). Because these stars are tidally locked, the apsidal line naturally marks such a "preferred" region on the stellar surface, i.e. the facing or the anti-facing hemispheres of the two components. Thus, preferred groupings of spots was not a complete surprise for the close binaries. However, active longitudes were later-on also discovered in single active stars by Jetsu et al. (1991). This made it clear that the phenomenon must be of magnetic origin rather than due to tidal forces, at least for the single stars.

It is now well established that some active stars exhibit rotationallymodulated light curves that remain stable in phase over long periods of time and then, more-or-less suddenly, switch phase by $180^{\circ}$, after which a time of light minimum becomes a time of light maximum and vice versa. This can happen repeatedly and we speak of a "flip-flop". The most noticeable examples are the very rapidly rotating single G-giants FK Comae (Jetsu et al. 1994) and possibly HD 199178 (Jetsu et al. 1990), and the RS CVn binary II Pegasii (Berdyugina et al. 1999, Rodonó et al. 2000). As for II Peg, the flip-flop has now been also seen in Doppler images of FK Comae, and it was demonstrated that the phase flips are caused by high-latitude spots (Korhonen et al. 2001, 2002). Detecting a single periodicity of the "flip-flop-flip" is not straightforward because the flip-flop appears to be present during some epochs, but then absent in some other epochs. This suggests some sort of oscillatory behavior. Korhonen et al. found a pseudo-periodicity of around $\approx 6$ yrs for FK Comae. 


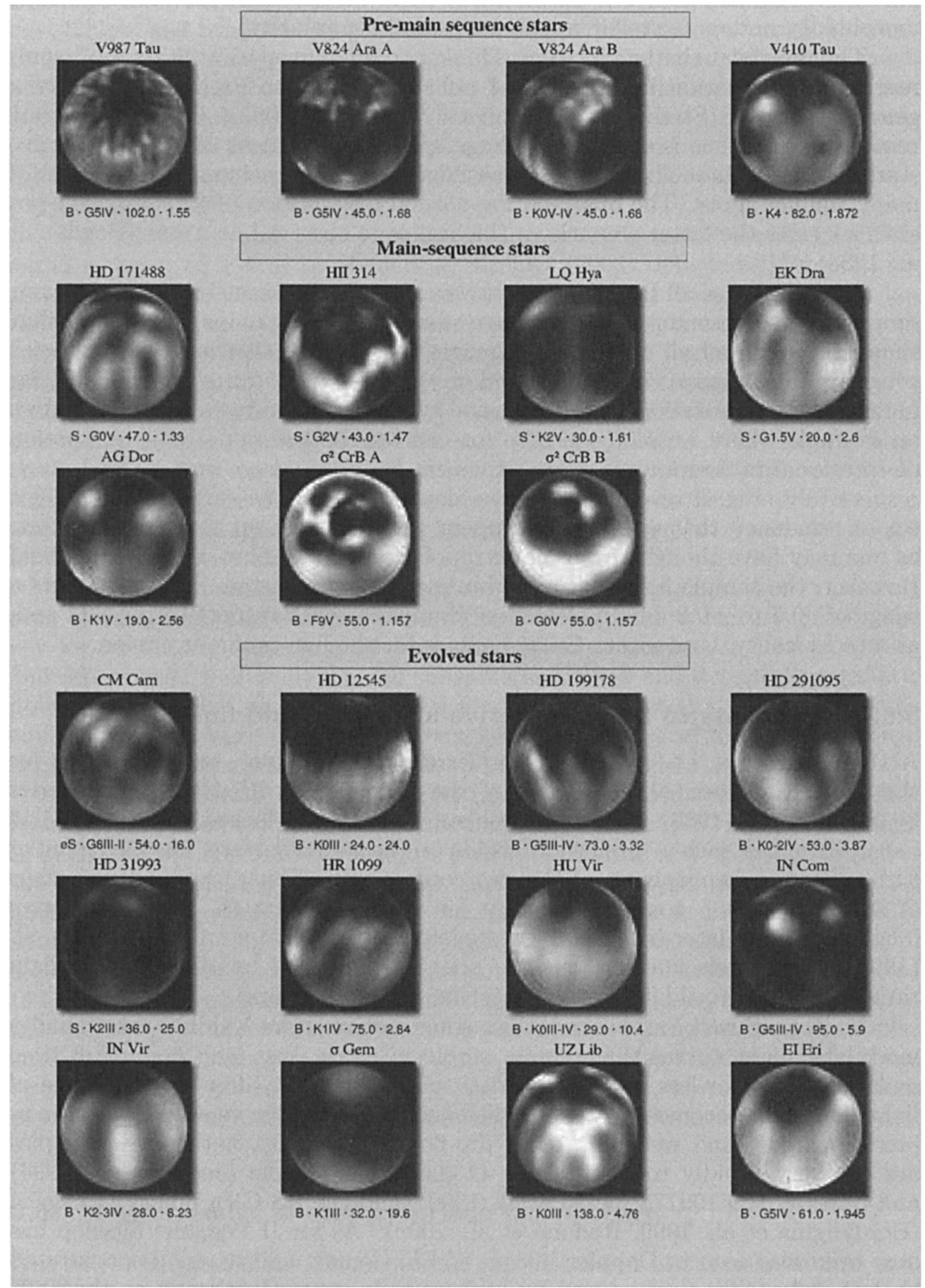

Figure 3. Results from the "Doppler imaging of stellar surface structure" program. The program has reached paper XXI in A\&A (Strassmeier, Pichler \& Weber 2003, on HD171488). 
Other active stars - binaries as well as single stars, pre-main-sequence as well as post-ms - show a remarkably stable phase coherence over a decade or so, sometimes with and sometimes without a phase flip; e.g., the K-giant of the RS CVn-type binary UZ Librae (Oláh et al. 2001), the single young K-dwarf LQ Hydrae (Berdyugina et al. 2002, Kövári et al. 2004), or the single weaklined T Tauri V410 Tau (Granzer et al. 2001). Maybe these targets simply have much longer flip-flop periods, and some periods without a periodicity?

Finally, Berdyugina \& Usoskin (2003) succeeded to detect active longitudes and the flip-flops also on the Sun. They analyzed 120 years of sunspot data and carried out the same analysis as for LQ Hydrae and found flip-flop periods of $3.8 \mathrm{yrs}$ and $3.65 \mathrm{yrs}$ for the northern and the southern hemisphere, respectively. This result is, for the first time, an example where a phenomenon was first detected on stars and then verified on the Sun. The stellar-solar connection!

One explanation of the flip-flop would be to have both axisymmetric and non-axisymmetric dynamo modes excited at the same time (Tuominen et al. 2002). They suggested that the two active longitudes would have different polarity and furthermore speculated that after a flip-flop event the polarities of the active longitudes would have changed. Just very recently, Elstner (2004) has produced a nice 3 -D simulation of an $\alpha \Omega$-dynamo where stationary, i.e. axisymmetric, and non-stationary, i.e. non-axisymmetric, solutions coexist and are being excited alternately. This model reproduces the flip-flop at high stellar latitudes (see www.aip.de/groups/MHD ).

\section{Summary}

After three decades of intense research with an average of 500 papers published per year, the solar-stellar connection now not only leads to new insights in classical fields like stellar evolution and coronal physics or new fields like planet hunting, but also faced us with some unexpected discoveries and conclusions (see the "cool-star history" review by Dupree 2003) . Firstly, the discovery of extra-solar planets around cool stars expanded the solar/stellar connection to one of a solar-system/stellar connection. Secondly, the fact that we had not found a real solar twin so far may indicate yet a disconnection (my guess though is that we have not searched deep enough yet). Thirdly, the dynamo-generated magnetic field and its transportation to the stellar surface is now recognized to be an important process and may affect normal stellar evolution.

Into the future, the next major progress will arrive from detailed studies of the stellar surface magnetic field with the new generation of instruments like, e.g., the PEPSI spectropolarimeter for the $2 \times 8.4 \mathrm{~m} \mathrm{LBT}$. A bit further into the future, the next milestone will be from space-based asteroseismology of solartype stars. Visionary space missions like the Stellar imager, which combines asteroseismology and interferometric imaging, will likely dominate in the twenties of this young century. The recent discovery that the host stars of extra-solar planets tend to be more metal rich paves the way for the ultimate goal of a "simple-minded" stellar astronomer, like "Give me a high-resolution spectrum of a star and I tell you if it has a planet". We'll see; now dig up your german dictionary: 
Wer alle seine Ziele erreicht, hat sie wahrscheinlich zu niedrig gewählt. ${ }^{3}$ (Herbert von Karajan)

Acknowledgments. I am grateful to the German Science Foundation for their support with grant STR645/1. and, of course, to Andrea Dupree and Arnold Benz for organizing the meeting. It is always an honour to acknowledge the collaboration with John Rice from Brandon University and with Thomas "Wooz" Granzer, Michi Weber, Heidi Korhonen, Igor Savanov and Günther Rüdiger from Potsdam. I also thank Dr. Rainer Arlt for providing Fig. 1.

\section{References}

Arlt, R., Hollerbach, G., \& Rüdiger, G., 2003, A\&A 401, 1087

Arlt, R., \& Rüdiger, G., 2003, www.aip.de/groups/MHD/

Baliunas, S. L., et al., 1985, ApJ 294, 310

Baliunas, S. L., et al., 1995, ApJ 438, 269

Barnes, S. A., 2003, ApJ 586, 464

Barnes, J., Cameron, A. C.,\& Donati, J.-F., et al. 2000, MNRAS 314, 162

Barrett, E. A., Boesgaard, A., King, J., \& Deliyannis, C., 2001, AAS 199, 5710 Basu, S., 1997, MNRAS 288, 572

Basu, S., et al., 2003, ApJ 591, 432

Berdyugina, S., Berdyugin, A., Ilyin, I.,\& Tuominen, I., 2001, in Garcia Lopez et al. (eds.), 11th Cool Stars, Stellar Systems and the Sun, PASPC 223, p. 1201

Berdyugina, S.,\& Berdyugin, A., et al., 2002, A\&A 350, 626

Berdyugina S., Pelt J.,\& Tuominen I., 2002, A\&A 394, 505

Berdyugina, S.,\& Usoskin I. G., 2002, A\&A 405, 1121

Bogdan, T. J., Gilman, P. A., Lerche, I.,\& Howard, R., 1988, ApJ 327, 451

Carpenter, K., et al., 2003, Stellar Imager, "Vision-Mission" proposal to NASA Cattaneo, F., 1999, ApJ 515, L39

Cayrel de Strobel, R., 1996, A\&A Rev. 7, 243

Cameron, A. C.,\& Donati, J.-F., 2002, MNRAS 329, L23

Charbonneau, P., et al., 1999, ApJ 527, 445

Delfosse, X., Forveille, T., Perrier, C.,\& Mayor, M., 1998, A\&A 331, 581

Donahue, R.,\& Baliunas, S. L., 1992, ApJ 393, L63

Donati, J.-F., 1999, MNRAS 302, 457

Donati, J.-F., Mengel, M.,\& Carter, B. D., 2000, MNRAS 316, 699

Donati, J.-F.,\& Cameron, A. C., 1997, MNRAS 291, 1

Donati, J.-F., Cameron, A. C.,\& Petit, P., 2003, MNRAS, in press

Dorren, J. D., \& Guinan; E. F., 1994, ApJ 448, 431

${ }^{3}$ Editors' translation: One who reaches all his goals has probably aimed too low. 
Drake, J. J., Stern, R. A., Stringfellow, G. S. et al., 1996, ApJ 469, 828

Dupree, A. K., 2003, in T. Ayres \& A. Brown (eds.), 12th Workshop on Cool Stars, Stellar Systems, and the Sun, origins.colorado.edu/cs12/

Eberhard, G.,\& Schwarzschild, K., 1913, ApJ 38, 292

Elstner, D., 2004, www.aip.de/groups/MHD/

Frazier, E. N., 1972, SoPh 24, 98

Freytag, B., Steffen, M.,\& Dorch, B., 2002, AN 323, 213

Fröhlich, H.-E., Kroll, P., Rüdiger, G., \& Strassmeier, K. G., 2004, A\&A, subm.

Gaidos, E. J., 1998, PASP 110, 1259

Gaidos, E. J.,\& Gonzales, G., 2002, New Astronomy 7, 211

Gaidos, E. J., Henry G. W.,\& Henry S. M., 2000, AJ 120, 1006

Granzer, T., 2002, AN 323, 395

Granzer, T., Reegen, P., \& Strassmeier, K. G., 2001, AN 322, 325

Gray, D. F., 1977, ApJ 211, 198

Gray, D. F., 1995, PASP 107, 120

Gray, D. F., \& Baliunas, S. L., 1997, ApJ 475, 303

Hackman, T., 2003, A\&A, submitted

Hackman, T., Jetsu, L.,\& Tuominen, I., 2001, A\&A 374, 171

Hall, D. S., et al., 1991, AJ 102, 1808

Hall, J. C. (ed.), 1997, Solar Analogs: Characteristics and Optimum Candidates, Lowell Obs., www.lowell.edu/users/jch/workshop/sa.html

Hardorp, J., 1978, A\&A 63, 383

Hartmann, L., et al., 1981, ApJ 249, 662

Henry, G. W., Eaton, J. A., Hamer, J., \& Hall, D.S., 1995, ApJS 97, 513

Henry, T. J., et al. 1997, AJ 114, 388

Henry, T. J., et al. 2002, AJ 123, 2002

Howard, R., \& Gilman, P. A., 1986, ApJ 307, 389

Huang, S. S., 1961, ApJ 133, 130

Jetsu, L., et al. 1990, A\&A 236, 423

Jetsu, L., et al. 1994, A\&A 282, L9

Jetsu, L., Pelt, J., Tuominen, I.,\& Nations, H. L., 1991, in IAU Coll. 130, I. Tuominen et al. (eds), The Sun and Cool Stars: Activity, Magnetism, Dynamos, Springer, p. 381

Jetsu, L., et al. 2000, A\&A 362, 223

Johns-Krull, C.,\& Hatzes, A. P., 1997, ApJ 487, 896

Johns-Krull, C.,\& Valenti, J. A., 1996, ApJ 459, L95

Kervella, P., et al., 2003, A\&A 404, 1087

Kitchatinov, L.,\& Rüdiger, G., 1999, A\&A 344, 911

Klessen, R., 2003, Habilitationsschrift, Univ. Potsdam

Korhonen, H., 2002, Ph-D thesis, Univ. of Oulu

Korhonen, H., Berdyugina, S. V., Strassmeier, K. G., Tuominen, I., 2001, A\&A 379, L30 
Korhonen, H., Berdyugina, S. V., Hackman, T., Ilyin, I., Strassmeier, K. G.,\& Tuominen, I., 2003, A\&A 379, L30

Korhonen, H., Berdyugina, S., \& Tuominen, I., 2002, A\&A 390, 179

Kővári, Zs., Strassmeier, K. G., Granzer, T., et al., 2004, A\&A, submitted

Kővári, Zs., Strassmeier, K. G., Bartus, J., et al., 2001, A\&A 373, 199

Küker, M., \& Stix, M., 2001, A\&A 366, 668

Lanza, A. F., et al., 2002, A\&A 386, 583

Lister, T. A., Collier Cameron, A.,\& Bartus, J., 1999, MNRAS 307, 685

Lockwood, G. W., Skiff B.,\& Radick, R., 1997, ApJ 485, 789

McCaughrean, M., et al., 2003, A\&A Lett., in press (astro-ph/0309256)

Messina, S., et al., 1999, A\&A 347, 249

Oláh, K., Strassmeier, K. G., 2002, AN 323, 361

Oláh, K., Strassmeier, K. G.,\& Granzer, T., 2002, AN 323, 453

Oláh, K., Jurcsik, J.,\& Strassmeier, K. G., 2003, A\&A 410, 685

Parker, E. H., 1955, ApJ 122, 293

Petit, P., Donati, J.-F., Wade, G. A., et al., 2003, MNRAS, in press

Reiners, A.,\& Schmitt, J., 2003, A\&A 398, 647

Rice, J. B., 2002, AN 323, 220

Rodonó, M., Lanza A.,\& Catalano, S., 1995, A\&A 301, 75

Rodonó, M., Messina, S., Lanza, A., et al., 2000, A\&A 358, 624

Rüdiger, G., \& Hollerbach, R., 2004, Magnetism in the Universe, Wiley-VCH, Berlin, in prep.

Saar, S. H., \& Brandenburg, A., 1999, ApJ 524, 295

Scholz, R.-D., et al., 2003, A\&A 398, L29

Schrijver, C. J., 1996, in IAU Symp. 176, Stellar Surface Structure, Strassmeier, K. G. \& Linsky, J. (eds.), Kluwer, p.1

Slettebak, A., 1949, ApJ 110, 498

Strassmeier, K. G., 1994, A\&A 281, 395

Strassmeier, K. G., 2002, AN 323, 309

Strassmeier, K. G., \& Bartus, J., 2000, A\&A, 354, 537

Strassmeier, K. G., \& Bartus, J., Kővári, Zs., et al., 1998, A\&A, 336, 587

Strassmeier, K. G., Hall, D. S., \& Henry, G. W., 1994, A\&A, 282, 535

Strassmeier, K. G., Kratzwald, L., \& Weber, M., 2003, A\&A, 408, 1103

Strassmeier, K. G., Lupinek, S., Dempsey, R.,\& Rice, J., 1999, A\&A 347, 212

Strassmeier, K. G., Pichler, T., Weber, M., \& Granzer, T. 2003, A\&A, 411, 595

Strassmeier, K. G., Washuettl, A.,\& Schwope, A. (eds.), 2002, AN 323, 155

Tuominen, J., 1942, ZfAp 21, 96

Tuominen, I., Berdyugina, S.,\& Korpi, M. J., 2002, AN 323, 367

Vogt, S.S., \& Hatzes, A.P., 1991, in IAU Coll. 130, I. Tuominen et al. (eds), The Sun and Cool Stars: Activity, Magnetism, Dynamos, Springer, p. 297

Vogt, S. S., \& Hatzes, A. P., 1996, in IAU Symp. 176, Stellar Surface Structure, Strassmeier K. G. \& Linsky J. (eds.), Kluwer, p.245 
Vogt, S. S., Hatzes, A. P., Misch, A.,\& Kürster, M., 1999, ApJS 121, 546

Vogt, S. S., \& Penrod, G. D., 1983, PASP 95, 565

Walker, G. A. H., et al., 2003, www.gemini.edu/

Washuettl, A., Strassmeier, K. G., Foing, B. H. et al., 2004, A\&A, in prep.

Weber, M., 2003, Ph-D thesis, Univ. Potsdam, in preparation

Weber, M., \& Strassmeier, K. G., 1998, A\&A 330, 1029

Weber, M., \& Strassmeier, K. G., 2001, A\&A 373, 974

Wedemeyer, S., Freytag, B., Steffen, M., Ludwig, H.-G., \& Holweger, H., 2003, AN 324,410

Zeilik, M., Elston, R., \& Henson, G., 1983, ApJ 88, 532

Ziegler, U., 2002, A\&A 386, 331

Ziegler, U., \& Rüdiger, G., 2003, A\&A 401, 433 

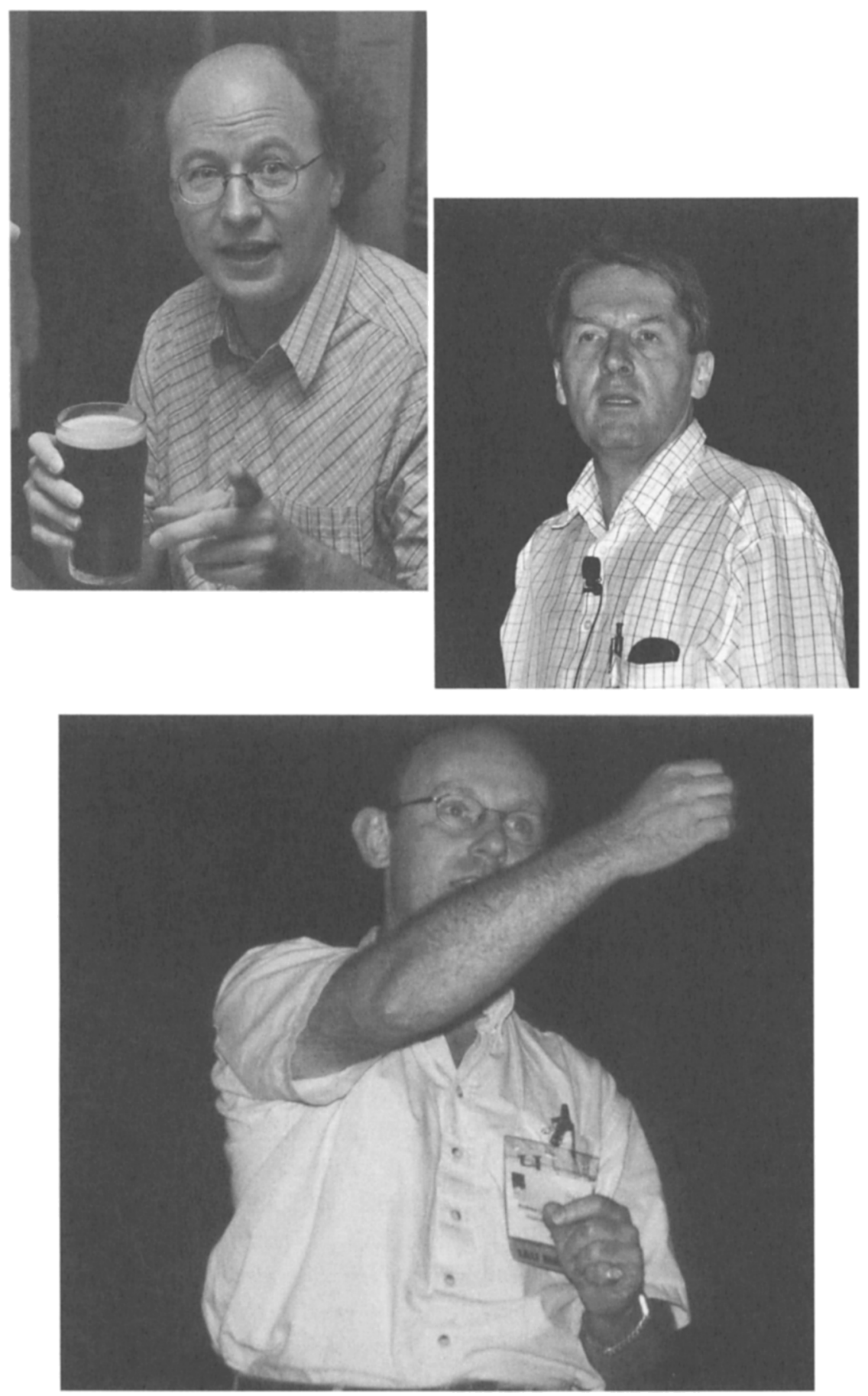